\title{
Foreword
}

\section{Modeling, Simulation, and Theory of Nanomechanical Materials Behavior}

\begin{abstract}
Although it is well appreciated that materials with nanometer-scale dimensions can have properties different than their bulk counterparts, specific observations continue to surprise and excite practitioners across many materials-related technologies. Even relatively well-known phenomena continue to evade complete elucidation of their underlying, fundamental mechanisms. However, significant progress continues to be made as researchers combine careful experimentation with validated modeling and theory. It is a very bright outlook for our understanding of nanoscale materials behavior.
\end{abstract}

Herein, a collection of articles is presented that demonstrates the power of simulation and theory to reveal the driving forces that determine the mechanical behavior of nanomaterials. In the first article, Salehinia and Medyanik explore the very foundation of plasticity in metals via atomic-scale simulations of vacancy behavior. The exploration of deformation mechanisms in metals is continued in the second article as Li et al. use finite-element quantized crystal plasticity simulations to study the influence of grain-scale distributions on critical strength in nanocrystalline metals. In the third article, Estrin et al. advance a new theory that accounts for image forces on dislocations - a necessary ingredient for properly describing dislocation nucleation in nanocrystalline metals. This trio of articles provides an excellent example of the multiscale, multiphysics understanding that must be brought to bear on nanomechanical behavior. Illustrating the power of biomimetic materials design and hierarchical structures, Garcia et al. use diatom algae to guide the creation of new silica structures. As they present in the fourth article in the series, atomistic simulations allow them to understand the fundamental mechanisms driving significant enhancements in deformability and toughness for a typically brittle material. The fifth article, by Johnson et al., presents the results from a multiscale model to describe the mechanisms behind the extreme piezoresistivity observed in silicone/nickel nanocomposites. The authors use their model to provide a refined interpretation of existing experimental results. The final article, by Greaney et al., presents the highly nonintuitive result that mechanical vibrations in carbon nanotubes decay faster for an initially larger excitation. The authors liken this to the Mpemba effect where relatively warmer water freezes more quickly than cooler water. They demonstrate, via a vibrational mode projection algorithm, the dissipation mechanisms that lead to this behavior and discuss the implications for general nanomechanical systems.

We hope the reader finds this collection of articles to be an excellent example of the capabilities of modeling and theory to reveal the fundamental mechanisms driving nanomechanical behavior in materials.

Symposium Organizers:

T. Buchheit

Sandia National Laboratories, Albuquerque, NM

L.H. Friedman

National Institute of Standards and Technology, Gaithersburg, MD

S. Medyanik

Washington State University, Pullman, WA

D. Spearot

University of Arkansas, Fayetteville, AK

E.B. Webb, III

Lehigh University, Bethlehem, PA

Sandia National Laboratories, Albuquerque, NM 